\title{
Capsule Commentary on Shortell et al., A Multilevel Analysis of Patient Engagement and Patient-Reported Outcomes in Primary Care Practices of Accountable Care Organizations
}

\author{
George L. Jackson, PhD, MHA ${ }^{1,2}$ \\ ${ }^{1}$ Center for Health Services Research in Primary Care, Durham Veterans Affairs Health Care System, Durham, NC, USA; ${ }^{2}$ Division of General Internal \\ Medicine, Duke University, Durham, NC, USA.
}

$\mathrm{J}$ Gen Intern Med 32(6):680

DOI: $10.1007 / \mathrm{s} 11606-017-4006-1$

(c) Society of General Internal Medicine 2017

- or almost two decades, the Wagner Chronic Care Model - $(\mathrm{CCM})$, with its principles also adopted in modern conceptualizations of the patient-centered medical home, has represented a roadmap for establishing systems for effective chronic illness care. ${ }^{1,2}$ The CCM postulates that optimal chronic illness management occurs when primary care teams have the decision and information technology resources needed to provide self-management support in collaboration with community resources. This system should lead to "productive interactions" between "prepared, proactive practice teams" and "informed, activated patients." Such interactions are hypothesized to result in better outcomes.

Shortell and colleagues addressed whether having primary care teams that reported a patient-centered culture (i.e., prepared, proactive clinicians) and highly activated patients was associated with better patient-reported health outcomes among a group of more than 2000 individuals with cardiovascular disease and/or diabetes treated at 16 primary care practices. ${ }^{3}$

Patients who reported that the organization of their primary care practice was more in line with the CCM, as measured by the Patient Assessment of Chronic Illness Care, ${ }^{4}$ had better mental and physical health outcomes. However, this association was mediated by the degree of patient activation, as measured by the Patient Activation Measure (PAM). ${ }^{5}$ In other words, the association between the primary care organizational structure and patient-reported outcomes appears to operate through the level of patient activation. While it was not a mediator of the association between primary care practice organization and patient-reported outcomes, having a primary care team reporting a patient-centered culture was also associated with better outcomes.

The frequently published diagram of the CCM has an arrow indicating that the impact of the organization of primary care on outcomes, at least in part, is through having activated patients. ${ }^{1}$ Shortell et al. provide additional evidence for this mechanism. They also provide additional evidence that differences in activation, as measured by the short version of the PAM, are associated with better selfreported patient health. While the causal direction of this association cannot be established with this cross-sectional study, the results suggest the potential utility of enhancing patient activation though self-management support and the use of the PAM as an outcome measure for efforts to enhance the provision of chronic illness care.

Corresponding Author: George L. Jackson, PhD, MHA; Center for Health Services Research in Primary CareDurham Veterans Affairs Health Care System, Durham, NC, USA (e-mail: george.l. jackson@duke.edu).

\section{Compliance with Ethical Standards:}

Conflict of Interest: The author has no conflicts of interest with this article.

Disclaimer: The views expressed in this Capsule Commentary are those of the author and do not reflect the position or policy of the Department of Veterans Affairs, United States government, or Duke University.

\section{REFERENCES}

1. Wagner EH. Chronic disease management: what will it take to improve care for chronic illness? Eff Clin Pract. 1998;1(1):2-4.

2. Jackson GL, Powers BJ, Chatterjee R, et al. The patient centered medical home. A systematic review. Ann Intern Med. 2013;158(3):169178.

3. Shortell SM, Poon BY, Ramsay PP, et al. A multilevel analysis of patient engagement and patient-reported outcomes in primary care practices of accountable care organizations. J Gen Intern Med. doi: 10.1007/s11606016-3980-z.

4. Gugiu PC, Coryn C, Clark R, Kuehn A. Development and evaluation of the short version of the patient assessment of chronic illness care instrument. Chronic Illn. 2009;5(4):268-276.

5. Hibbard JH, Mahoney ER, Stockard J, Tusler M. Development and testing of a short form of the patient activation measure. Health Serv Res. 2005;40(6 Pt 1):1918-1930.

Published online February 8, 2017 